\title{
Femtosecond Charge Density Modulations in Photoexcited CuWO
}

Yohei Uemura, $\triangle$ Ahmed S. M. Ismail, $\triangle$ Sang Han Park, Soonnam Kwon, Minseok Kim, Yasuhiro Niwa, Hiroki Wadati, Hebatalla Elnaggar, Federica Frati, Ties Haarman, Niko Höppel, Nils Huse, Yasuyuki Hirata, Yujun Zhang, Kohei Yamagami, Susumu Yamamoto, Iwao Matsuda, Tetsuo Katayama, Tadashi Togashi, Shigeki Owada, Makina Yabashi, Uufuk Halisdemir, Gertjan Koster, Toshihiko Yokoyama, Bert M. Weckhuysen, and Frank M. F. de Groot*

Cite This: J. Phys. Chem. C 2021, 125, 7329-7336

Read Online

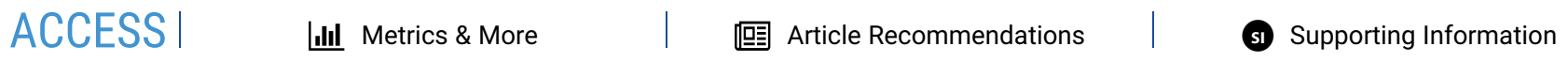

ABSTRACT: Copper tungstate $\left(\mathrm{CuWO}_{4}\right)$ is an important semiconductor with a sophisticated and debatable electronic structure that has a direct impact on its chemistry. Using the PAL$\mathrm{XFEL}$ source, we study the electronic dynamics of photoexcited $\mathrm{CuWO}_{4}$. The $\mathrm{Cu} \mathrm{L}_{3} \mathrm{X}$-ray absorption spectrum shifts to lower energy upon photoexcitation, which implies that the photoexcitation process from the oxygen valence band to the tungsten conduction band effectively increases the charge density on the $\mathrm{Cu}$ atoms. The decay time of this spectral change is $400 \mathrm{fs}$ indicating that the increased charge density exists only for a very short time and relaxes electronically. The initial increased charge density gives rise to a structural change on a time scale longer than 200 ps.

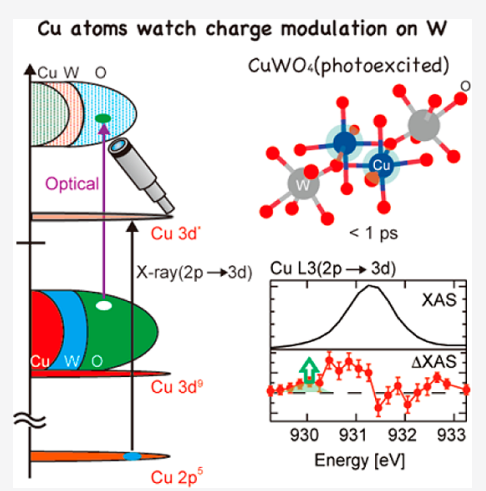

\section{INTRODUCTION}

Copper tungstate $\left(\mathrm{CuWO}_{4}\right)$ is an important functional material that has gained a lot of attention in materials science because of its small optical band gap and electrical conductivity. In particular, it is a promising candidate for photocatalysis ${ }^{1}$ and a potential photoelectrode for the water-splitting reaction. ${ }^{2-6}$ $\mathrm{CuWO}_{4}$ has a triclinic distorted wolframite-type structure due to the Jahn-Teller distortion around the $\mathrm{Cu}^{2+}$ ions. ${ }^{7}$ It is known as an n-type semiconductor and its valence band is believed to be composed mainly of oxygen $2 \mathrm{p}$ states that are hybridized with tungsten and copper s, p, and d states. ${ }^{8,9}$ The conduction band contains mainly W 5d states hybridized with the oxygen $2 p$ states. In addition, it has been claimed that the single $\mathrm{Cu} 3 \mathrm{~d}$ hole contributes to the bottom of the conduction band, which shifts the conduction band minimum in $\mathrm{CuWO}_{4}$ downward compared to $\mathrm{WO}_{3} \cdot{ }^{10-14}$ However, the origin of charge carriers and their behavior in $\mathrm{CuWO}_{4}$ are not well understood due to the scarcity of the experimental studies that probe the relaxation dynamics of the optically excited $\mathrm{Cu}$ electronic states, particularly in the fs/ps time domain. ${ }^{15,16}$ Therefore, we believe that understanding the ultrafast electronic transitions in $\mathrm{CuWO}_{4}$ in this time domain could provide a clearer insight on the nature of the electronic structure of $\mathrm{CuWO}_{4}$, which is highly debatable in literature $8,10,11,14,17$

In this work, we conducted the first investigation of the ultrafast charge carrier dynamics in $\mathrm{CuWO}_{4}$ using time-resolved optical and X-ray spectroscopies in the $100 \mathrm{fs}$ to $200 \mathrm{ps}$ time scale with a focus on the role of the copper $3 \mathrm{~d}$ states in the charge carrier transport process. Time-resolved X-ray absorption spectroscopy (XAS) provides detailed information on the ultrafast charge carrier dynamics and recombination in several metal oxide semiconductors. ${ }^{18-20}$ Charge carrier dynamics of water splitting semiconductors $\left(\mathrm{TiO}_{2},{ }^{21-23} \alpha\right.$ - $\mathrm{Fe}_{2} \mathrm{O}_{3},{ }^{24-27}$ $\mathrm{BiVO}_{4}{ }^{28,29} \mathrm{WO}_{3},{ }^{30-32} \mathrm{ZnO},{ }^{33}$ and $\mathrm{CsPbBr}_{3}$ and $\mathrm{CsPb}(\mathrm{ClBr})_{3}$ perovskites ${ }^{34}$ ) were successfully observed using ultrafast XAS. Herein, we present transient $\mathrm{Cu} \mathrm{L}_{3}$ XAS studies on the photoexcited state of $\mathrm{CuWO}_{4} \cdot \mathrm{Cu} \mathrm{L} \mathrm{L}_{3} \mathrm{XAS}$ is attributed to electron transition from $\mathrm{Cu} 2 \mathrm{p}$ orbitals to $\mathrm{Cu} 3 \mathrm{~d}$ orbitals, which reflects the chemical state of the $\mathrm{Cu}$ atoms directly. The optical laser excitation triggers a transition from the oxygen $2 p$ valence band to the $\mathrm{W} 5 \mathrm{~d}$ conduction band. ${ }^{8}$ The empty $\mathrm{Cu} 3 \mathrm{~d}$ state is not active in the optical transition and, as such, plays the role of a spectator state. During the X-ray excitation process, an electron is excited to the $\mathrm{Cu} 3 \mathrm{~d}$ band and one can observe its reaction to the optical excitation. This role as a spectator state makes it attractive to study its response to the optical excitation, in order to determine the model of the electronic structure and the dynamics in electronic states of $\mathrm{CuWO}_{4}$.

Received: November 23, 2020

Revised: $\quad$ March 1, 2021

Published: March 26, 2021 


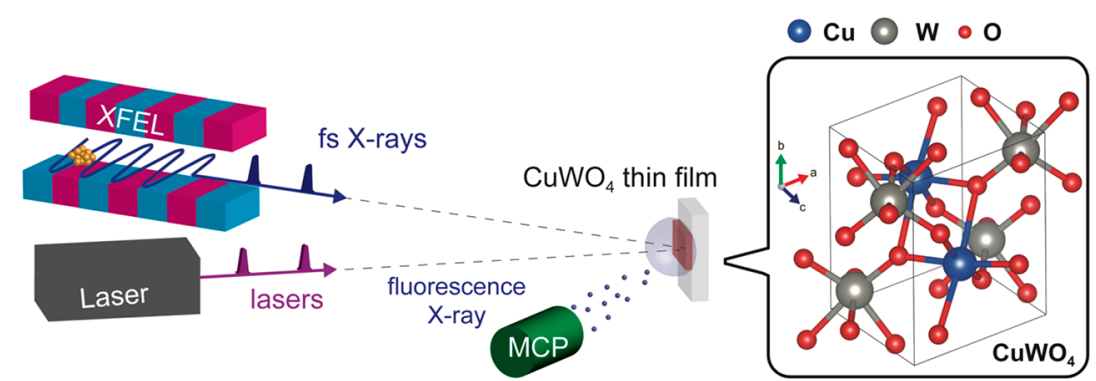

Figure 1. Time-resolved X-ray absorption spectroscopy (XAS) on $\mathrm{CuWO}_{4}$ : experimental setup of the pump-probe XAS in PAL-XFEL and the crystal structure of $\mathrm{CuWO}_{4}$.
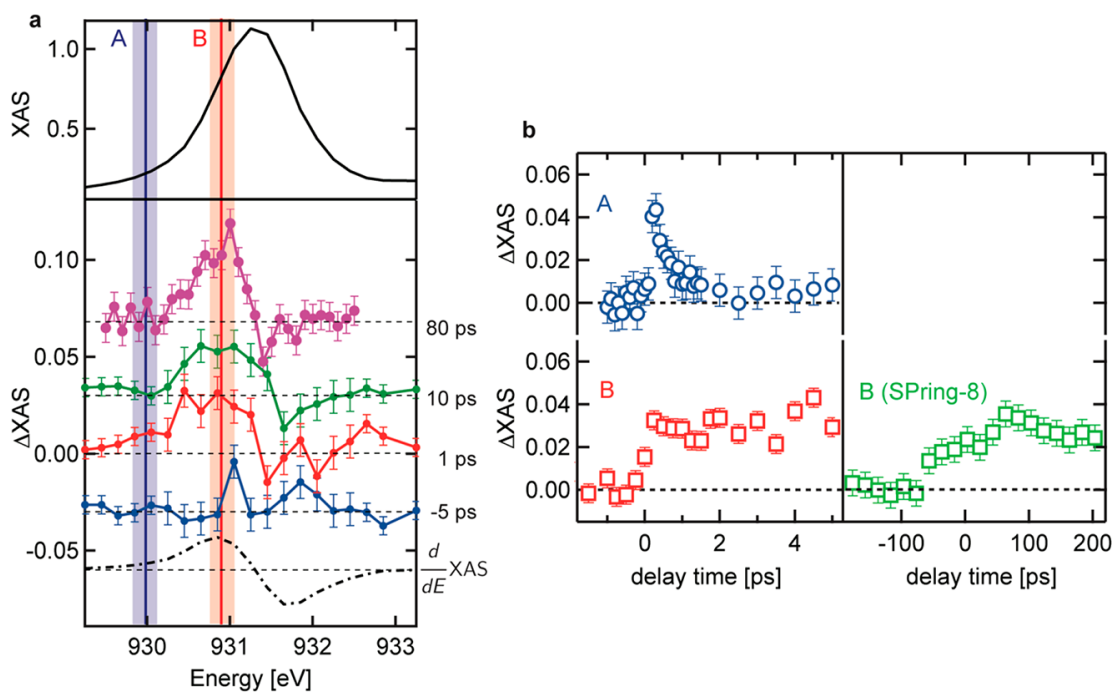

Figure 2. (a) Normalized $\mathrm{Cu} \mathrm{L}_{3} \mathrm{XAS}$ (top panel) and the transient X-ray absorption spectroscopy (XAS) data of Cu $\mathrm{L}_{3}$ edge in $\mathrm{CuWO}_{4}$ at several delay times $\left(-5,1,10\right.$, and $80 \mathrm{ps}$ with respect to $-10 \mathrm{ps}$ ) (bottom panel). The transient XAS spectra were normalized according to the reference $\mathrm{Cu} \mathrm{L}_{3} \mathrm{XAS}$. (b) Kinetic traces of the $\mathrm{Cu} \mathrm{L}_{3}$ edge at peaks $\mathrm{A}$ and $\mathrm{B}$ measured at PAL-XFEL and the kinetic trace of $\mathrm{Cu} \mathrm{L}_{3}$ edge XAS at peak B for longer delay times measured at SPring-8. The intensities were normalized according to the reference $\mathrm{Cu} \mathrm{L}_{3} \mathrm{XAS}$. The error bars for the transient XAS were estimated from the standard deviation of the transient XAS at a negative delay time. Due to shot-by-shot variations of the X-ray intensity, there remains a point-bypoint error that can be larger than the standard deviation. The error bars for the kinetic traces were estimated from the standard deviation of the unpumped XAS intensity.

\section{EXPERIMENTS}

The $\mathrm{CuWO}_{4}$ sample was a polycrystalline thin film with a thickness of $50 \mathrm{~nm}$ synthesized using pulsed laser deposition (PLD) supported on a conductive glass substrate (more details of the sample preparation and physical characterizations are described in the Supporting Information). Pump-probe $\mathrm{Cu} \mathrm{L}_{3}$ XAS experiments were conducted in the soft X-ray spectroscopy and scattering (SSS) beamline at PAL-XFEL. ${ }^{35-37}$ A simple illustration of the experimental setup is shown in Figure 1. The sample was placed at the focal point of the $\mathrm{X}$-rays (the focal size of the X-rays was less than $50 \mu \mathrm{m}(H) \times 50 \mu \mathrm{m}(V))$. The 400 nm optical pump laser was transferred to the focal position of the X-rays. The focal size of the optical laser was $\sim 210 \mu \mathrm{m}(H) \times$ $210 \mu \mathrm{m}(V)$. XAS signals were obtained using a michrochannel plate (MCP) detector. A more detailed experimental setup is shown in Figure S3. For the observation of transient XAS at longer delay time ( $>80 \mathrm{ps}$ ), pump-probe experiments were conducted at BL07LSU, SPring- $8 .^{38}$ The detailed experimental setup at SPring- 8 as well as other experimental details (optical pump-probe experiments and static $\mathrm{Cu}$ K-edge XAS) are described in the Supporting Information.

The X-ray intensity fluctuates shot-by-shot in PAL-XFEL, and some X-ray shots have too large or too small intensities to obtain
XAS signals. Therefore, such X-ray shots were removed from data sets since they worsen the signal to noise ratio $(S / N)$. Details of analyzing the X-ray data to calculate XAS are described in Supporting Information. In addition to the shot-byshot fluctuations, variations in the intensity of XFEL pulses were observed over several hours, which might affect the XAS signal and result in artificial differential spectra. To avoid these artifacts, the XAS at each delay time and those at the reference were measured in sequence. That is to say, a reference XAS spectrum at a delay time of -10 ps, i.e., an excitation laser pulse arrives at the sample $10 \mathrm{ps}$ after an X-ray pulse arrives, was measured for each measurement in order to calculate a difference spectrum for each delay time. Each difference XAS spectrum $(\triangle \mathrm{XAS})$ at a delay $(\Delta t)$ was calculated by subtracting $\mathrm{XAS}$ at $-10 \mathrm{ps}$ from $\mathrm{XAS}$ at $\Delta t$, i.e.,

$$
\Delta \mathrm{XAS}=\operatorname{XAS}(\Delta t[\mathrm{ps}])-\operatorname{XAS}(\Delta t=-10 \mathrm{ps})
$$

At SPring-8, the laser pump repetition rate was set to half of the $\mathrm{X}$-ray probe repetition rate. XAS signals with or without laser shots were accumulated consecutively. 

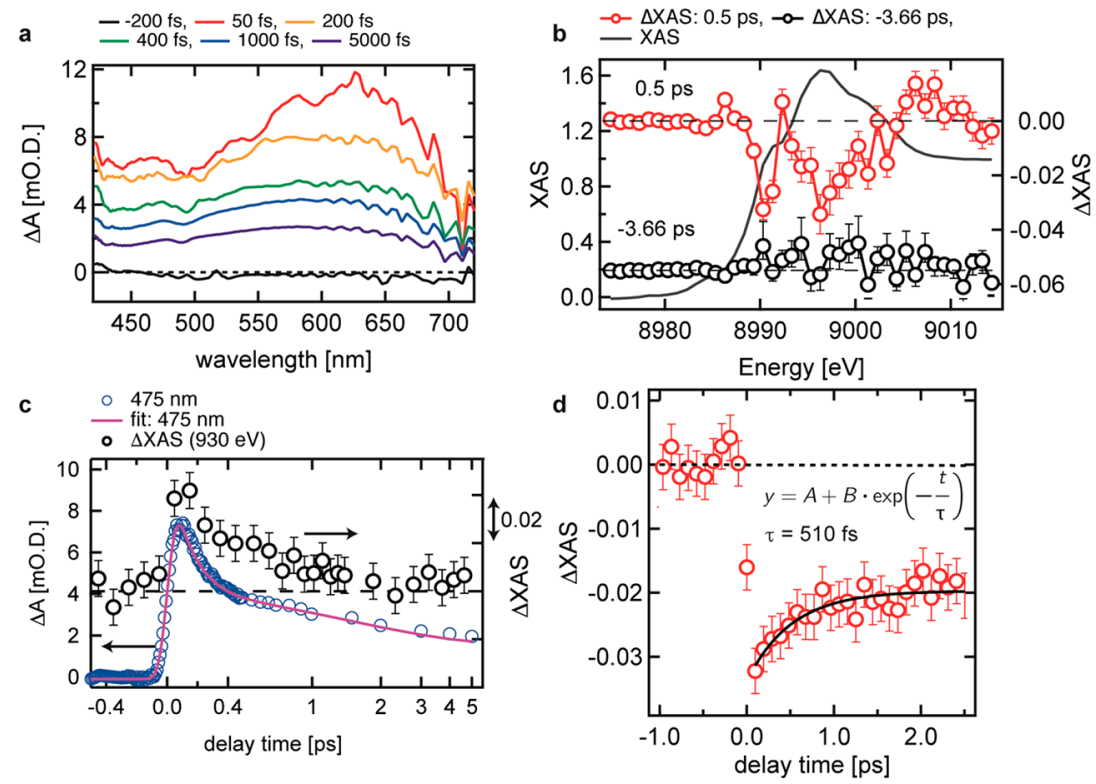

Figure 3. (a) Transient optical absorbance of $\mathrm{CuWO}_{4}$ from 420 to $720 \mathrm{~nm}$. (b) Transient $\mathrm{Cu}$ K-edge XAS of $\mathrm{CuWO}_{4}$ before photoexcitation $(-3.66$ ps) and after photoexcitation $(0.5 \mathrm{ps})$. The difference spectra were normalized according to the Cu K-edge XAS in the optical ground state. (c) Kinetic trace of the transient optical absorbance of $\mathrm{CuWO}_{4}$ at $475 \mathrm{~nm}$. (d) Kinetic trance of transient $\mathrm{Cu}$ K-edge XAS of CuWO $\mathrm{Cu}_{4}$ at $8980 \mathrm{eV}$. The intensity was normalized according to the difference XAS shown in part $b$.

\section{RESULTS}

3.1. Transient $\mathrm{Cu} \mathrm{L}_{3}$ XAS of $\mathrm{CuWO}_{4}$. The transient XAS spectra at several delays are displayed in Figure 2. The difference XAS spectra at delays of $-5 \mathrm{ps}, 1 \mathrm{ps}$, and $10 \mathrm{ps}$ were measured at PAL-XFEL, whereas the difference XAS spectrum at a delay of 80 ps was measured at SPring-8. After optical excitation, one observes an absorption increase at energies below the static $\mathrm{L}_{3}$ edge maximum of $931.4 \mathrm{eV}$ and in addition there is a minor absorption decrease at energies above the $\mathrm{L}_{3}$ edge maximum. We note that the observed spectral changes do not agree with the first derivative of $\mathrm{Cu} \mathrm{L}_{3}$ XAS (dashed line). Figure $2 \mathrm{~b}$ (right panels) shows the kinetic traces of the $\mathrm{L}_{3}$ edge at $930.0 \mathrm{eV}$ (position A) and $930.8 \mathrm{eV}$ (position B). The kinetic trace at position $B$ shows a rise related to the time resolution of the experiment followed by a constant intensity increase over the whole range of 5 ps. The absorption change at position $B$ was measured up to $200 \mathrm{ps}$, but at a delay time of $200 \mathrm{ps}$, and was not recovered to its original ground state value at this time delay (Figure 2b, SPring-8). On the other hand, the kinetic trace of peak $\mathrm{A}(930.0 \mathrm{eV})$ decays on a much shorter time scale, where the kinetic constant of this fast process was found to be $400 \pm$ 160 fs (Figure S4).

3.2. Pump-Probe Optical Spectroscopy and Transient $\mathrm{Cu}$ K-Edge XAS of $\mathrm{CuWO}_{4}$. A similar fast decay process was also observed by optical pump-probe spectroscopy shown in Figure $3 \mathrm{a}$ and Figure $3 \mathrm{c}$ and by pump-probe $\mathrm{Cu}$ K-edge XAS conducted at SPring-8 angstrom compact free electron laser (SACLA $)^{39-43}$ shown in Figure 3b and Figure 3d. In the optical spectroscopy study, a broad transient absorption was observed between 420 and $720 \mathrm{~nm}$. This broad absorption should be mainly attributed to excited electrons distributed in the conduction band. Since excited electrons have different energies and transition to different final states, a broad absorption can be observed after photoexcitation. As described in Figure S2, there were four time constants extracted from a rate-equation model. The $\mathrm{Cu}$ K-edge XAS after photoexcitation is interpreted by the shift of the $\mathrm{Cu}$ K-edge XAS to lower energy. This result agrees with the increase of the electron density at the $\mathrm{Cu}$ sites. A fast kinetic process was observed at $8990.3 \mathrm{eV}$, which decays by a delay of 2 ps. The kinetic constant of this process was estimated to be $510 \pm 150$ fs. This value is consistent with the kinetic constant estimated by $\mathrm{Cu} \mathrm{L}_{3}$ edge within the error limits. This excess decrease of the X-ray intensity could reflect the excess charge density at the $\mathrm{Cu}$ atoms in agreement with the $\mathrm{Cu} \mathrm{L}_{3}$ edge data.

3.3. Interpretation of the Transient $\mathrm{Cu}_{3}$ XAS. We first note that the peak position of $\mathrm{Cu}^{2+}$ in the $\mathrm{Cu} \mathrm{L}_{3} \mathrm{XAS}$ is lower than that of both $\mathrm{Cu}^{1+}$ and $\mathrm{Cu}^{3+}$ (for example, refs 44-46), which is in contrast to all other transition metal ions. The reason is that the ground state of $\mathrm{Cu}^{2+}$ is $3 \mathrm{~d}^{9}$ and the only allowed final state is $2 \mathrm{p}^{5} 3 \mathrm{~d}^{10}$; hence there is only a single peak observed at the $\mathrm{L}_{3}$ edge. The peak position of $\mathrm{Cu}^{3+}$ appears at higher energy position due to the larger effective charge of the copper ion. Also, the first $\mathrm{L}_{3}$ peak of $\mathrm{Cu}^{1+}$ appears at higher energy since the $3 \mathrm{~d}$ band of $\mathrm{Cu}^{1+}$ is full and the intensity is given by the transition to a $4 \mathrm{~s}$ orbital that forms an exciton. ${ }^{47}$ Interestingly neither a change to $\mathrm{Cu}^{1+}$ or $\mathrm{Cu}^{3+}$ will allow a peak at an energy lower than $\mathrm{Cu}^{2+}$. In other words, the spectral change at $930.0 \mathrm{eV}$ cannot be assigned to change of the valence state of the copper ion.

Instead, we assign this feature to $\mathrm{Cu}^{2+}$ that has a modified local electronic structure. The optical excitation moves an electron from the oxygen $2 \mathrm{p}$ valence band to the tungsten $5 \mathrm{~d}$ conduction band, while $\mathrm{Cu}^{2+}$ is essentially a spectator ion. Since the charge density of the copper $\mathrm{s}$ and $\mathrm{p}$ states is larger in the $\mathrm{W} 5 \mathrm{~d}$ conduction band than in the oxygen $2 p$ valence band, the effective charge density on the $\mathrm{Cu}^{2+}$ ions will increase creating a $\mathrm{Cu}^{2+} 3 \mathrm{~d}^{9}$ ion that has enhanced electron density, which will shift the $L_{3}$ edge to lower energy. This enhanced charge density is contained within band states with copper $\mathrm{s}$ and $\mathrm{p}$ character, and being a delocalized band state, this extra charge will rapidly delocalize, faster than the time constant of $400 \mathrm{fs}$. The change in charge distribution can affect the vibrational potential in the excited system due to electron-lattice coupling. ${ }^{48}$ Under such situation, low-frequency modes move toward this new origin 
a
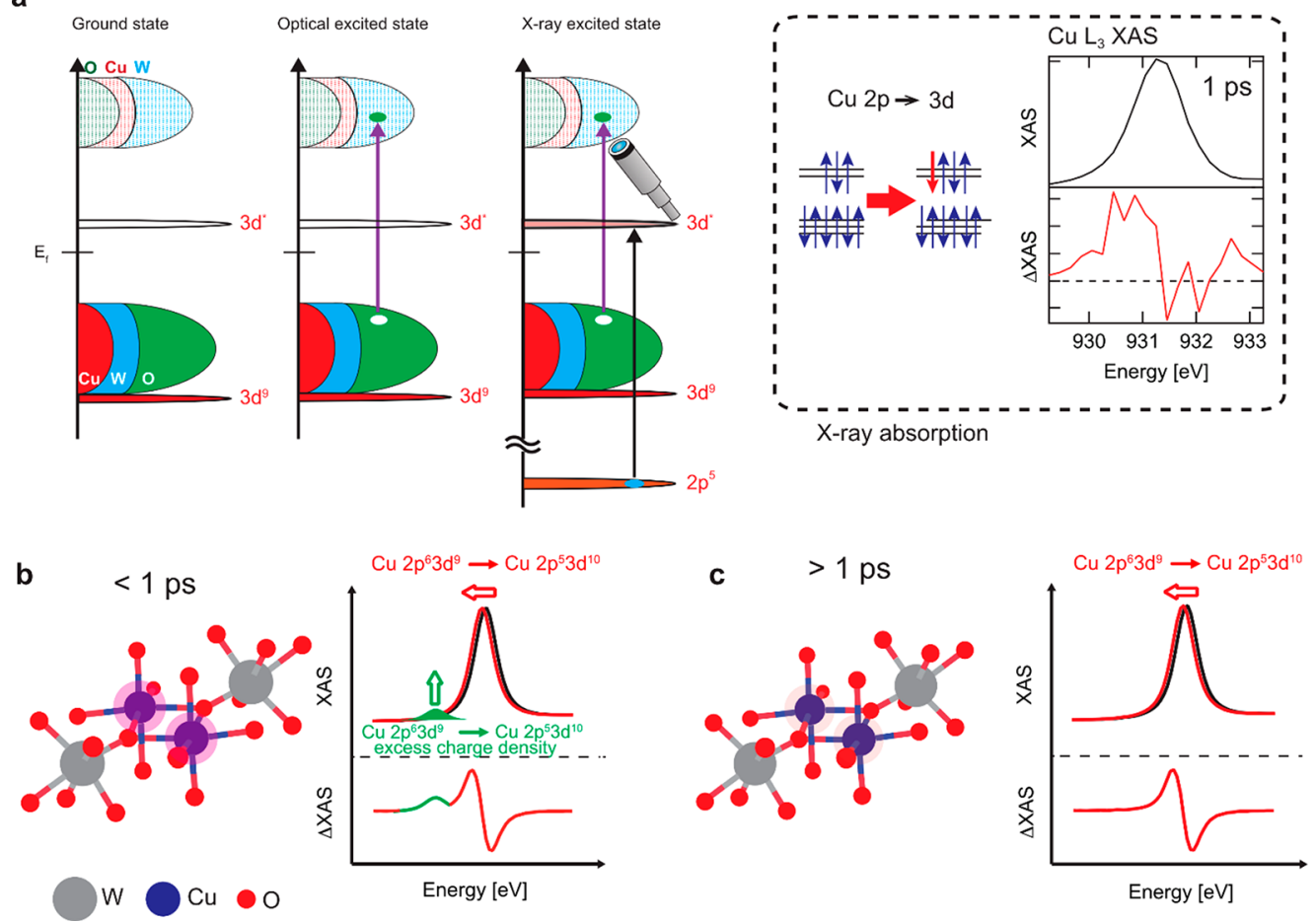

Figure 4. (a) A sketch that explains the optical and $\mathrm{X}$-ray excitation processes in $\mathrm{CuWO}_{4}$ and the $\mathrm{Cu}_{3}$-edge transient $\mathrm{XAS}$ spectra at a delay time of 10 ps. Illustrations of the charge density at $\mathrm{Cu}$ sites in $\mathrm{CuWO}_{4}$ after photoexcitation below a delay of $1 \mathrm{ps}(\mathrm{b})$ and above a delay of 1 ps (c).

with the modified interatomic distances and made additional modification to charge distribution. The experiment thus uses the copper $\mathrm{L}_{3}$ edge spectrum as an indicator of the local charge density changes (and phonon modes) on the copper site following optical laser excitation.

3.4. Photoexcitation Dynamics of $\mathrm{CuWO}_{4}$. Two main changes were observed by transient $\mathrm{Cu} \mathrm{L}_{3}$ XAS upon optical excitation of $\mathrm{CuWO}_{4}$ : (i) An initial photoexcited state is created by optical excitation, creating enhanced charge density at the copper sites, decaying with a time constant of 400 fs. The enhanced charge density is related to copper $\mathrm{s}$ and $\mathrm{p}$ states, and the valence remains $\mathrm{Cu}^{2+}$. (ii) The photoexcited state undergoes a decay process to form a metastable state that has a lifetime of more than 200 ps. The metastable state has increased charge density with respect to the optical ground state but reduced with respect to the initial excited state. This metastable state must involve structural reorganization of atoms related to a polaronic state while the valence remains $\mathrm{Cu}^{2+}$. It should be noted that local structural changes can also shift the $\mathrm{Cu} \mathrm{L}_{3}$ edge XAS to lower energy; i.e., if the $\mathrm{Cu}-\mathrm{O}$ distance is elongated by $10 \%$, calculations show that the $\mathrm{Cu} \mathrm{L}_{3}$ XAS shifts by $\sim 0.2 \mathrm{eV}{ }^{49}$ The local structural change affects more directly the spectral features of the $\mathrm{Cu}$ K-edge XAS in higher energy, which is visible at $~ 9000$ $\mathrm{eV}$ at delays of 2.3 and 8.3 ps (see Figure S5). Local structural changes are related to the formation of polaronic states. Regarding the polaron state formation in $\mathrm{CuWO}_{4}$, Hoang et al. suggested that an electron hole should be more localized on the $\mathrm{O}$ atoms while an electron would be more localized on metal sites. ${ }^{14}$ Considering their results, we conclude that the effective negative charge on the $\mathrm{O}$ atoms would be decreased by $+\delta$ while the effective positive charge on $\mathrm{W}$ atoms and $\mathrm{Cu}$ atoms would also decrease by $-\delta$, reducing the charge difference and thereby increasing the bond length.

\section{DISCUSSION}

4.1. Model of the Electronic Structure of $\mathrm{CuWO}_{4}$. As mentioned above, the electronic structure of $\mathrm{CuWO}_{4}$ is still an open question and there have been models suggested by several authors. For instance, Tian et al. ${ }^{8}$ reported that the bottom of the conduction band of $\mathrm{CuWO}_{4}$ comes from $\mathrm{Cu} 3 \mathrm{~d}$ orbitals while the top of the valence band is formed by $\mathrm{Cu} 3 \mathrm{~d}$ orbitals and $\mathrm{O} 2 \mathrm{p}$ orbitals. Hoang et al. ${ }^{14}$ pointed out that $\mathrm{O} 2 \mathrm{p}$ orbitals are dominant in the valence band of $\mathrm{CuWO}_{4}$ while its conduction band is formed mainly by $\mathrm{Cu} 3 \mathrm{~d}$ orbitals and $\mathrm{W} 5 \mathrm{~d}$ orbitals. Khyzhun et al. ${ }^{11}$ suggested that the conduction band of $\mathrm{CuWO}_{4}$ is a W 5d-like state while its valence band is an O 2 p-like state and they also assume the existence of $\mathrm{Cu}^{3+} \cdot{ }^{10}$ Considering the previous reports and our transient XAS results, we assume a combined electronic structure of $\mathrm{WO}_{3}$-type structure with an oxygen $2 \mathrm{p}$ valence band and a W $5 \mathrm{~d}$ conduction band. The $3 \mathrm{~d}-$ electrons of the $\mathrm{Cu}^{2+}$ ion contribute to the oxygen $2 \mathrm{p}$ valence band, while its occupied $3 \mathrm{~d}$ electrons are positioned at lower energies, in line with all other $\mathrm{Cu}^{2+}$ oxides that are charge transfer insulators. ${ }^{17}$

In order to discuss the electronic structure of $\mathrm{CuWO}_{4}$ in detail, we first introduce the electronic structure of the binary parent oxides $\mathrm{WO}_{3}$ and $\mathrm{CuO}$. $\mathrm{WO}_{3}$ is a semiconductor with a valence band mainly composed of oxygen $2 p$ character. The oxygen $2 \mathrm{p}$ state hybridizes strongly with the tungsten $5 \mathrm{~d}, 6 \mathrm{~s}$, and $6 \mathrm{p}$ states that yield a considerable amount of $\mathrm{W}$ character in the valence band. The conduction band consists of the antibonding combinations of oxygen $2 \mathrm{p}$ states with tungsten $5 \mathrm{~d}, 6 \mathrm{~s}$, and $6 \mathrm{p}$ states. For simplicity, this could be called the tungsten $5 \mathrm{~d}$ band, but it also contains significant contributions of tungsten $s$ and $p$ character. The electronic structure of $\mathrm{CuO}$ is described as a charge transfer insulator, ${ }^{17}$ where the $\mathrm{Cu} 3 \mathrm{~d}$ states are split between the lower Hubbard band below the oxygen $2 p$ band and the empty upper Hubbard band that has one hole state. ${ }^{50}$ 
$\mathrm{CuWO}_{4}$ can be considered as a combination of both oxides. The (top of the) valence band contains mainly oxygen $2 p$ character mixed with $\mathrm{W} 5 \mathrm{~d}$ and $\mathrm{sp}$ states plus $\mathrm{Cu} 3 \mathrm{~d}$ and sp states. The conduction band is dominated by W $5 \mathrm{~d}$ but also contains oxygen $2 \mathrm{p}$ and copper $3 \mathrm{~d}$ and sp states. In between, the upper Hubbard band of $\mathrm{Cu} 3 \mathrm{~d}$ sits in the band gap (see Figure 4a).

4.2. The 400 fs Metastable Charge Density Modulation. The laser excitation of $\mathrm{CuWO}_{4}$ involves a transition from the oxygen $2 \mathrm{p}$ valence band to the tungsten $5 \mathrm{~d}$ conduction band. This creates a ligand hole plus an electron in the W $5 \mathrm{~d}$ band. The $\mathrm{Cu} 3 \mathrm{~d}$ states are spectators to this laser excitation since the $\mathrm{Cu}$ $3 \mathrm{~d}$ states are not influenced by the photoexcitation as described (section 3.3). The electronic structure of the copper sites is however affected because the excited electron in the conduction band has partly copper $4 \mathrm{~s} / 4 \mathrm{p}$ character. This additional charge at the $\mathrm{Cu}$ site will decrease the binding energy of the $2 \mathrm{p}$ core states, following the general rule that the lower is the number of electrons at a metal site (i.e., a higher valency) the higher is its binding energy. Thus, the laser excitation creates a "spectator" copper site with a lower binding energy of its $2 p$ core states.

The $\mathrm{Cu} 2 \mathrm{p}$ XAS spectrum of $\mathrm{CuWO}_{4}$ shows essentially one sharp peak and at higher energy some weaker intensity. The sharp peak is related to the $3 \mathrm{~d}^{9} \rightarrow 2 \mathrm{p}^{5} 3 \mathrm{~d}^{10}$ transition. The laser excited $\mathrm{CuWO}_{4}$ sample will also have a single $3 \mathrm{~d}^{9} \rightarrow 2 \mathrm{p}^{5} 3 \mathrm{~d}^{10}$ transition, but due to the reduced binding energy of the $2 \mathrm{p}$ core state, this transition will be shifted to lower excitation energy. The small absorption appears due to this shifted $3 \mathrm{~d}^{9} \rightarrow 2 \mathrm{p}^{5} 3 \mathrm{~d}^{10}$ transition (the green small peak in Figure $4 \mathrm{~b}$ ). In other words, we can track the charge density change of the $\mathrm{Cu}$ spectator state by measuring the time evolution of the transient signal at an excitation energy below the copper $\mathrm{L}_{3}$ edge (see Figure $4 \mathrm{~b}$ and Figure $4 \mathrm{c}$ ). The $\mathrm{Cu}$ state with the modified charge density has a lifetime of only $400 \mathrm{fs}$. The $400 \mathrm{~nm}$ laser excites the electrons $\sim 3.1 \mathrm{eV}$ up in energy, and with a band gap of $2.3 \mathrm{eV}$ they are excited in the bottom $0.8 \mathrm{eV}$ of the conduction band. The corresponding modified charge density can lose energy via carrier cooling and electronic decay channels to the lowest states of the conduction band. Potentially this electronic picture could be modified by phonons affecting the charge density on copper via a changing $\mathrm{Cu}-\mathrm{O}$ distance.

4.3. Effect on Photocatalysis. The observations above provide insights on the photocatalytic behavior of $\mathrm{CuWO}_{4}$ compared to $\mathrm{WO}_{3}$ and $\mathrm{CuO}$. Adding $\mathrm{Cu}$ to $\mathrm{WO}_{3}$ modifies the band gap of the system, making it capable of absorbing optical light in the visible range of the solar spectrum which enhances its photocatalytic performance. The system still requires a large overpotential to drive photocatalytic processes such as water splitting. This is possibly due to the formation of a polaronic state. The initial optical excitation creates a modified charge density that is viewed from the $\mathrm{Cu}$ site in our study. After the decay of the original optical excitation within $400 \mathrm{fs}$, the system does not return to its original state, which is a clear indication of the formation of a polaronic state. It is believed that polarons are formed due to lattice distortion around the light-absorbing atom, resulting in trapping and hence reducing the mobility of electrons, which effects the photocatalytic efficiency of the system. The formation of this polaronic state has been observed in several metal oxide systems. ${ }^{26,51,52}$

Furthermore, it is important to note that in order to create an effective photocatalyst, one needs to optimize the system of photon capture, which is mainly governed by the band gap. The latter is determined by the overlap between tungsten and oxygen. Copper remains $\mathrm{Cu}^{2+}$ throughout the whole process of the optical excitation and the 400 fs decay. In that sense the $\mathrm{Cu}$ state is described as a spectator that is not redox active but rather creates a better (electronic and structural) environment for the $\mathrm{WO}_{3}$ system that receives the optical photon.

\section{CONCLUDING REMARKS}

In summary, by tracking a $50 \mathrm{fs}, 3.1 \mathrm{eV}$ optical laser excitation in $\mathrm{CuWO}_{4}$ via a pump-probe delayed X-ray excitation $1.0 \mathrm{eV}$ before the $\mathrm{Cu} \mathrm{L}_{3}$ edge, we have identified an increased charge density at the $\mathrm{Cu}$ site that has a decay time of $400 \mathrm{fs}$. This fast decay implies that the modified charge density decays electronically via carrier cooling to a longer-lived electronic excited state. It would be very interesting to investigate if this type of charge modulation with decay times of a few hundred femtoseconds is a general phenomenon in transition metal oxides and if this phenomenon is correlated with photocatalytic activity that can be modified by sample preparation and by the parameters in the laser excitation.

\section{ASSOCIATED CONTENT}

\section{Supporting Information}

The Supporting Information is available free of charge at https://pubs.acs.org/doi/10.1021/acs.jpcc.0c10525.

Physical characterizations of the $\mathrm{CuWO}_{4}$ thin films (SEM, XRD, XPS, AFM, UV-Vis spectrum, and a Tauc plot), details of the transient optical spectroscopy, experimental details of transient XAS spectroscopy (PAL-XFEL, SPring- 8 and SACLA), and supplemental results of transient XAS at SACLA (PDF)

\section{AUTHOR INFORMATION}

\section{Corresponding Author}

Frank M. F. de Groot - Inorganic Chemistry and Catalysis, Debye Institute for Nanomaterials Science, Utrecht University, 3584 CG Utrecht, The Netherlands; ㅇ orcid.org/00000002-1340-2186; Email: F.M.F.deGroot@uu.nl

\section{Authors}

Yohei Uemura - Inorganic Chemistry and Catalysis, Debye Institute for Nanomaterials Science, Utrecht University, 3584 CG Utrecht, The Netherlands; Institute for Catalysis, Hokkaido University, Sapporo, Hokkaido 001-0021, Japan; (1) orcid.org/0000-0003-3164-7168

Ahmed S. M. Ismail - Inorganic Chemistry and Catalysis, Debye Institute for Nanomaterials Science, Utrecht University, 3584 CG Utrecht, The Netherlands; 이이.org/00000002-2282-1665

Sang Han Park - PAL-XFEL, Pohang Accelerator Laboratory, Pohang, Gyeongbuk 37673, South Korea

Soonnam Kwon - PAL-XFEL, Pohang Accelerator Laboratory, Pohang, Gyeongbuk 37673, South Korea

Minseok Kim - PAL-XFEL, Pohang Accelerator Laboratory, Pohang, Gyeongbuk 37673, South Korea

Yasuhiro Niwa - Photon Factory, Institute for Materials Structure Science, KEK, Tsukuba 305-0801, Japan

Hiroki Wadati - Institute for Solid State Physics, University of Tokyo, Kashiwa, Chiba 277-8581, Japan; Graduate School of Material Science, University of Hyogo, Kamigori, Hyogo 6781297, Japan

Hebatalla Elnaggar - Inorganic Chemistry and Catalysis, Debye Institute for Nanomaterials Science, Utrecht University, 
3584 CG Utrecht, The Netherlands; (1) orcid.org/00000002-4223-4054

Federica Frati - Inorganic Chemistry and Catalysis, Debye Institute for Nanomaterials Science, Utrecht University, 3584 CG Utrecht, The Netherlands

Ties Haarman - Inorganic Chemistry and Catalysis, Debye Institute for Nanomaterials Science, Utrecht University, 3584 CG Utrecht, The Netherlands

Niko Höppel - Department of Physics and Center for FreeElectron Laser Science, University of Hamburg, 22761 Hamburg, Germany

Nils Huse - Department of Physics and Center for Free-Electron Laser Science, University of Hamburg, 22761 Hamburg, Germany; 이이이.org/0000-0002-3281-7600

Yasuyuki Hirata - Institute for Solid State Physics, University of Tokyo, Kashiwa, Chiba 277-8581, Japan

Yujun Zhang - Institute for Solid State Physics, University of Tokyo, Kashiwa, Chiba 277-8581, Japan; 이이.org/00000003-4892-1100

Kohei Yamagami - Institute for Solid State Physics, University of Tokyo, Kashiwa, Chiba 277-8581, Japan

Susumu Yamamoto - Institute for Solid State Physics, University of Tokyo, Kashiwa, Chiba 277-8581, Japan; (1) orcid.org/0000-0002-6116-7993

Iwao Matsuda - Institute for Solid State Physics, University of Tokyo, Kashiwa, Chiba 277-8581, Japan; 이이이.org/00000002-2118-9303

Tetsuo Katayama - JASRI, Sayo-cho, Hyogo 679-5198, Japan; RIKEN SPring-8 Center, Sayo-cho, Hyogo 679-5148, Japan

Tadashi Togashi - JASRI, Sayo-cho, Hyogo 679-5198, Japan; RIKEN SPring-8 Center, Sayo-cho, Hyogo 679-5148, Japan

Shigeki Owada - JASRI, Sayo-cho, Hyogo 679-5198, Japan; RIKEN SPring-8 Center, Sayo-cho, Hyogo 679-5148, Japan

Makina Yabashi - RIKEN SPring-8 Center, Sayo-cho, Hyogo 679-5148, Japan

Uufuk Halisdemir - Faculty of Science and Technology and MESA+ Institute for Nanotechnology, University of Twente, 7500 AE Enschede, The Netherlands

Gertjan Koster - Faculty of Science and Technology and MESA + Institute for Nanotechnology, University of Twente, 7500 AE Enschede, The Netherlands; 10 orcid.org/0000-0001-54787329

Toshihiko Yokoyama - Institute for Molecular Science, Okazaki 444-8585, Japan; 이이.org/0000-0003-01617216

Bert M. Weckhuysen - Inorganic Chemistry and Catalysis, Debye Institute for Nanomaterials Science, Utrecht University, 3584 CG Utrecht, The Netherlands; 이이.org/00000001-5245-1426

Complete contact information is available at:

https://pubs.acs.org/10.1021/acs.jpcc.0c10525

\section{Author Contributions}

$\triangle$ Y.U. and A.S.M.I. contributed equally to this work.

\section{Notes}

The authors declare no competing financial interest.

\section{ACKNOWLEDGMENTS}

This work was financially supported by the European Research Council (ERC) under the European Union's Horizon 2020 Research and Innovation Programme (Grant Agreement 340279), The Netherlands Center for Multiscale Catalytic
Energy Conversion (MCEC), a Gravitation Program from The Netherlands Organisation for Scientific Research (NWO), a grant for collaborative research in the Institute for Catalysis, Hokkaido University (Grant 18A1005), a Grant-in-Aid for Scientific Research (A) (Grant 15H02173, JSPS), and a basic science research program funded by the Ministry of Education of Korea (Grants NRF-2020R1A2C1007416 and 2018R1D1A1B07046676). N. Huse and N. Höppel acknowledge funding by the collaborative research center SFB 925 of the German Science Foundation (DFG), project 170620586. The experiment at SACLA was performed with an approval of Japan Synchrotron Radiation Research Institute (JASRI; Proposal 2018A8049). We thank Prof. Thomas Elsasser (Max-Born Institute/Humboldt Universität zu Berlin) and Prof. Kiyotaka Asakura (Hokkaido University) for useful comments and suggestions.

\section{REFERENCES}

(1) Momeni, M. M. Fabrication of copper decorated tungsten oxidetitanium oxide nanotubes by photochemical deposition technique and their photocatalytic application under visible light. Appl. Surf. Sci. 2015, $357,160-166$.

(2) Lhermitte, C. R.; Bartlett, B. M. Advancing the Chemistry of $\mathrm{CuWO}_{4}$ for Photoelectrochemical Water Oxidation. Acc. Chem. Res. 2016, 49, 1121-1129.

(3) Valenti, M.; Dolat, D.; Biskos, G.; Schmidt-Ott, A.; Smith, W. A. Enhancement of the Photoelectrochemical Performance of $\mathrm{CuWO}_{4}$ Thin Films for Solar Water Splitting by Plasmonic Nanoparticle Functionalization. J. Phys. Chem. C 2015, 119, 2096-2104.

(4) Yourey, J. E.; Pyper, K. J.; Kurtz, J. B.; Bartlett, B. M. Chemical Stability of $\mathrm{CuWO}_{4}$ for Photoelectrochemical Water Oxidation. J. Phys. Chem. C 2013, 117, 8708-8718.

(5) Hill, J. C.; Ping, Y.; Galli, G. A.; Choi, K.-S. Synthesis, photoelectrochemical properties, and first principles study of n-type $\mathrm{CuW}_{1-\mathrm{x}} \mathrm{Mo}_{\mathrm{x}} \mathrm{O}_{4}$ electrodes showing enhanced visible light absorption. Energy Environ. Sci. 2013, 6, 2440-2446.

(6) Benko, F. A.; MacLaurin, C. L.; Koffyberg, F. P. $\mathrm{CuWO}_{4}$ and $\mathrm{Cu}_{3} \mathrm{WO}_{6}$ as anodes for the photoelectrolysis of water. Mater. Res. Bull. 1982, 17, 133-136.

(7) Schofield, P. F.; Knight, K. S.; Redfern, S. A. T.; Cressey, G. Distortion Characteristics Across the Structural Phase Transition in $\left(\mathrm{Cu}_{1-\mathrm{x}} \mathrm{Zn}_{\mathrm{x}}\right) \mathrm{WO}_{4}$. Acta Crystallogr., Sect. B: Struct. Sci. 1997, 53, 102112.

(8) Tian, C. M.; Jiang, M.; Tang, D.; Qiao, L.; Xiao, H. Y.; Oropeza, F. E.; Hofmann, J. P.; Hensen, E. J. M.; Tadich, A.; Li, W.; et al. Elucidating the electronic structure of $\mathrm{CuWO}_{4}$ thin films for enhanced photoelectrochemical water splitting. J. Mater. Chem. A 2019, 7, 11895-11907.

(9) Kihlborg, L.; Gebert, E. CuWO 4 , a distorted Wolframite-type structure. Acta Crystallogr., Sect. B: Struct. Crystallogr. Cryst. Chem. 1970, 26, 1020-1026.

(10) Khyzhun, O. Y.; Strunskus, T.; Cramm, S.; Solonin, Y. M. Electronic structure of $\mathrm{CuWO}_{4}$ : XPS, XES and NEXAFS studies. J. Alloys Compd. 2005, 389, 14-20.

(11) Khyzhun, O. Y.; Bekenev, V. L.; Solonin, Y. M. First-principles calculations and $\mathrm{X}$-ray spectroscopy studies of the electronic structure of $\mathrm{CuWO}_{4}$. J. Alloys Compd. 2009, 480, 184-189.

(12) Lalić, M. V.; Popović, Z. S.; Vukajlović, F. R. Ab initio study of electronic, magnetic and optical properties of $\mathrm{CuWO}_{4}$ tungstate. Comput. Mater. Sci. 2011, 50, 1179-1186.

(13) Gaillard, N.; Chang, Y.; Braun, A.; DeAngelis, A. Copper Tungstate $\left(\mathrm{CuWO}_{4}\right)$-Based Materials for Photoelectrochemical Hydrogen Production. MRS Proc. 2012, 1446, 31-36.

(14) Hoang, K.; Oh, M.; Choi, Y. Electronic structure, polaron formation, and functional properties in transition-metal tungstates. RSC Adv. 2018, 8, 4191-4196. 
(15) Hirst, J.; Müller, S.; Peeters, D.; Sadlo, A.; Mai, L.; Reyes, O. M.; Friedrich, D.; Mitoraj, D.; Devi, A.; Beranek, R.; et al. Comparative Study of Photocarrier Dynamics in CVD-deposited $\mathrm{CuWO}_{4}, \mathrm{CuO}$, and $\mathrm{WO}_{3}$ Thin Films for Photoelectrocatalysis. Z. Phys. Chem. (Muenchen, Ger.) 2020, 234, 699-717.

(16) Peeters, D.; Mendoza Reyes, O.; Mai, L.; Sadlo, A.; Cwik, S.; Rogalla, D.; Becker, H. W.; Schütz, H. M.; Hirst, J.; Müller, S.; et al. CVD-grown copper tungstate thin films for solar water splitting. J. Mater. Chem. A 2018, 6, 10206-10216.

(17) Zaanen, J.; Sawatzky, G. A.; Allen, J. W. Band gaps and electronic structure of transition-metal compounds. Phys. Rev. Lett. 1985, 55, 418-421.

(18) Kraus, P. M.; Zürch, M.; Cushing, S. K.; Neumark, D. M.; Leone, $\mathrm{S}$. R. The ultrafast X-ray spectroscopic revolution in chemical dynamics. Nat. Rev. Chem. 2018, 2, 82-94.

(19) Chergui, M.; Collet, E. Photoinduced Structural Dynamics of Molecular Systems Mapped by Time-Resolved X-ray Methods. Chem. Rev. (Washington, DC, U. S.) 2017, 117, 11025-11065.

(20) Milne, C. J.; Penfold, T. J.; Chergui, M. Recent experimental and theoretical developments in time-resolved X-ray spectroscopies. Coord. Chem. Rev. 2014, 277-278, 44-68.

(21) Obara, Y.; Ito, H.; Ito, T.; Kurahashi, N.; Thürmer, S.; Tanaka, H.; Katayama, T.; Togashi, T.; Owada, S.; Yamamoto, Y.-i.; et al. Femtosecond time-resolved X-ray absorption spectroscopy of anatase $\mathrm{TiO}_{2}$ nanoparticles using XFEL. Struct. Dyn. 2017, 4, No. 044033.

(22) Santomauro, F. G.; Lübcke, A.; Rittmann, J.; Baldini, E.; Ferrer, A.; Silatani, M.; Zimmermann, P.; Grübel, S.; Johnson, J. A.; Mariager, S. O.; et al. Femtosecond X-ray absorption study of electron localization in photoexcited anatase $\mathrm{TiO}_{2}$. Sci. Rep. 2015, 5, 14834.

(23) Rittmann-Frank, M. H.; Milne, C. J.; Rittmann, J.; Reinhard, M.; Penfold, T. J.; Chergui, M. Mapping of the Photoinduced Electron Traps in $\mathrm{TiO}_{2}$ by Picosecond X-ray Absorption Spectroscopy. Angew. Chem., Int. Ed. 2014, 53, 5858-5862.

(24) Ismail, A. S. M.; Uemura, Y.; Park, S. H.; Kwon, S.; Kim, M.; Elnaggar, H.; Frati, F.; Niwa, Y.; Wadati, H.; Hirata, Y.; et al. Direct observation of the electronic states of photoexcited hematite with ultrafast 2p3d X-ray absorption spectroscopy and resonant inelastic Xray scattering. Phys. Chem. Chem. Phys. 2020, 22, 2685-2692.

(25) Leshchev, D.; Harlang, T. C. B.; Fredin, L. A.; Khakhulin, D.; Liu, Y.; Biasin, E.; Laursen, M. G.; Newby, G. E.; Haldrup, K.; Nielsen, M. M.; et al. Tracking the picosecond deactivation dynamics of a photoexcited iron carbene complex by time-resolved X-ray scattering. Chem. Sci. 2018, 9, 405-414.

(26) Carneiro, L. M.; Cushing, S. K.; Liu, C.; Su, Y.; Yang, P.; Alivisatos, A. P.; Leone, S. R. Excitation-wavelength-dependent small polaron trapping of photoexcited carriers in $\alpha-\mathrm{Fe}_{2} \mathrm{O}_{3}$. Nat. Mater. 2017, $16,819-825$.

(27) Vura-Weis, J.; Jiang, C.-M.; Liu, C.; Gao, H.; Lucas, J. M.; de Groot, F. M. F.; Yang, P.; Alivisatos, A. P.; Leone, S. R. Femtosecond $\mathrm{M}_{2,3}$-Edge Spectroscopy of Transition-Metal Oxides: Photoinduced Oxidation State Change in $\alpha-\mathrm{Fe}_{2} \mathrm{O}_{3}$. J. Phys. Chem. Lett. 2013, 4, 36673671.

(28) Uemura, Y.; Kido, D.; Koide, A.; Wakisaka, Y.; Niwa, Y.; Nozawa, S.; Ichiyanagi, K.; Fukaya, R.; Adachi, S.-i.; Katayama, T.; et al. Capturing local structure modulations of photoexcited $\mathrm{BiVO}_{4}$ by ultrafast transient XAFS. Chem. Commun. 2017, 53, 7314-7317.

(29) Ravensbergen, J.; Abdi, F. F.; van Santen, J. H.; Frese, R. N.; Dam, B.; van de Krol, R.; Kennis, J. T. M. Unraveling the Carrier Dynamics of $\mathrm{BiVO}_{4}$ : A Femtosecond to Microsecond Transient Absorption Study. J. Phys. Chem. C 2014, 118, 27793-27800.

(30) Koide, A.; Uemura, Y.; Kido, D.; Wakisaka, Y.; Takakusagi, S.; Ohtani, B.; Niwa, Y.; Nozawa, S.; Ichiyanagi, K.; Fukaya, R.; et al. Photoinduced anisotropic distortion as the electron trapping site of tungsten trioxide by ultrafast $\mathrm{W} \mathrm{L}_{1}$-edge $\mathrm{X}$-ray absorption spectroscopy with full potential multiple scattering calculations. Phys. Chem. Chem. Phys. 2020, 22, 2615-2621.

(31) Uemura, Y.; Kido, D.; Wakisaka, Y.; Uehara, H.; Ohba, T.; Niwa, Y.; Nozawa, S.; Sato, T.; Ichiyanagi, K.; Fukaya, R.; et al. Dynamics of Photoelectrons and Structural Changes of Tungsten Trioxide Observed by Femtosecond Transient XAFS. Angew. Chem., Int. Ed. 2016, 55, $1364-1367$.

(32) Uemura, Y.; Uehara, H.; Niwa, Y.; Nozawa, S.; Sato, T.; Adachi, S.; Ohtani, B.; Takakusagi, S.; Asakura, K. In Situ Picosecond XAFS Study of an Excited State of Tungsten Oxide. Chem. Lett. 2014, 43, 977-979.

(33) Penfold, T. J.; Szlachetko, J.; Santomauro, F. G.; Britz, A.; Gawelda, W.; Doumy, G.; March, A. M.; Southworth, S. H.; Rittmann, J.; Abela, R.; et al. Revealing hole trapping in zinc oxide nanoparticles by time-resolved X-ray spectroscopy. Nat. Commun. 2018, 9, 478.

(34) Santomauro, F. G.; Grilj, J.; Mewes, L.; Nedelcu, G.; Yakunin, S.; Rossi, T.; Capano, G.; Al Haddad, A.; Budarz, J.; Kinschel, D.; et al. Localized holes and delocalized electrons in photoexcited inorganic perovskites: Watching each atomic actor by picosecond X-ray absorption spectroscopy. Struct. Dyn. 2017, 4, No. 044002.

(35) Jang, H.; Kim, H.-D.; Kim, M.; Park, S. H.; Kwon, S.; Lee, J. Y.; Park, S.-Y.; Park, G.; Kim, S.; Hyun, H.; et al. Time-resolved resonant elastic soft $\mathrm{x}$-ray scattering at Pohang Accelerator Laboratory X-ray Free Electron Laser. Rev. Sci. Instrum. 2020, 91, No. 083904.

(36) Park, S. H.; Kim, M.; Min, C.-K.; Eom, I.; Nam, I.; Lee, H.-S.; Kang, H.-S.; Kim, H.-D.; Jang, H. Y.; Kim, S.; et al. PAL-XFEL soft Xray scientific instruments and $\mathrm{X}$-ray optics: First commissioning results. Rev. Sci. Instrum. 2018, 89, No. 055105.

(37) Kang, H.-S.; Min, C.-K.; Heo, H.; Kim, C.; Yang, H.; Kim, G.; Nam, I.; Baek, S. Y.; Choi, H.-J.; Mun, G.; et al. Hard X-ray free-electron laser with femtosecond-scale timing jitter. Nat. Photonics 2017, 11, $708-713$.

(38) Takubo, K.; Yamamoto, K.; Hirata, Y.; Yokoyama, Y.; Kubota, Y.; Yamamoto, S.; Yamamoto, S.; Matsuda, I.; Shin, S.; Seki, T.; et al. Capturing ultrafast magnetic dynamics by time-resolved soft x-ray magnetic circular dichroism. Appl. Phys. Lett. 2017, 110, 162401.

(39) Katayama, T.; Nozawa, S.; Umena, Y.; Lee, S.; Togashi, T.; Owada, S.; Yabashi, M. A versatile experimental system for tracking ultrafast chemical reactions with X-ray free-electron lasers. Struct. Dyn. 2019, 6, No. 054302.

(40) Katayama, T.; Hirano, T.; Morioka, Y.; Sano, Y.; Osaka, T.; Owada, S.; Togashi, T.; Yabashi, M. X-ray optics for advanced ultrafast pump-probe X-ray experiments at SACLAThis article will form part of a virtual special issue on X-ray free-electron lasers. J. Synchrotron Radiat. 2019, 26, 333-338.

(41) Katayama, T.; Owada, S.; Togashi, T.; Ogawa, K.; Karvinen, P.; Vartiainen, I.; Eronen, A.; David, C.; Sato, T.; Nakajima, K.; et al. A beam branching method for timing and spectral characterization of hard X-ray free-electron lasers. Struct. Dyn. 2016, 3, No. 034301.

(42) Tono, K.; Togashi, T.; Inubushi, Y.; Sato, T.; Katayama, T.; Ogawa, K.; Ohashi, H.; Kimura, H.; Takahashi, S.; Takeshita, K.; et al. Beamline, experimental stations and photon beam diagnostics for the hard x-ray free electron laser of SACLA. New J. Phys. 2013, 15, No. 083035.

(43) Ishikawa, T.; Aoyagi, H.; Asaka, T.; Asano, Y.; Azumi, N.; Bizen, T.; Ego, H.; Fukami, K.; Fukui, T.; Furukawa, Y.; et al. A compact X-ray free-electron laser emitting in the sub-ångström region. Nat. Photonics 2012, 6, 540-544.

(44) Huang, M. J.; Deng, G.; Chin, Y. Y.; Hu, Z.; Cheng, J. G.; Chou, F. C.; Conder, K.; Zhou, J. S.; Pi, T. W.; Goodenough, J. B.; et al. Determination of hole distribution in $\mathrm{Sr}_{14-\mathrm{x}} \mathrm{Ca}_{\mathrm{x}} \mathrm{Cu}_{24} \mathrm{O}_{41}$ using soft $\mathrm{x}$-ray absorption spectroscopy at the $\mathrm{Cu} \mathrm{L}_{3}$ edge. Phys. Rev. B: Condens. Matter Mater. Phys. 2013, 88, No. 014520.

(45) Choudhury, D.; Rivero, P.; Meyers, D.; Liu, X.; Cao, Y.; Middey, S.; Whitaker, M. J.; Barraza-Lopez, S.; Freeland, J. W.; Greenblatt, M.; et al. Anomalous charge and negative-charge-transfer insulating state in cuprate chain compound $\mathrm{KCuO}_{2}$. Phys. Rev. B: Condens. Matter Mater. Phys. 2015, 92, 201108.

(46) Wang, Y.; Lany, S.; Ghanbaja, J.; Fagot-Revurat, Y.; Chen, Y. P.; Soldera, F.; Horwat, D.; Mücklich, F.; Pierson, J. F. Electronic structures of $\mathrm{Cu}_{2} \mathrm{O}, \mathrm{Cu}_{4} \mathrm{O}_{3}$, and $\mathrm{CuO}$ : $\mathrm{A}$ joint experimental and theoretical study. Phys. Rev. B: Condens. Matter Mater. Phys. 2016, 94, 245418 . 
(47) Grioni, M.; Goedkoop, J. B.; Schoorl, R.; de Groot, F. M. F.; Fuggle, J. C.; Schäfers, F.; Koch, E. E.; Rossi, G.; Esteva, J. M.; Karnatak, R. C. Studies of copper valence states with $\mathrm{Cu} \mathrm{L}_{3} \mathrm{x}$-ray absorption spectroscopy. Phys. Rev. B: Condens. Matter Mater. Phys. 1989, 39, $1541-1545$.

(48) Ruiz-Fuertes, J.; Segura, A.; Rodríguez, F.; Errandonea, D.; SanzOrtiz, M. N. Anomalous High-Pressure Jahn-Teller Behavior in $\mathrm{CuWO}_{4}$. Phys. Rev. Lett. 2012, 108, 166402.

(49) Ye, X.; Schmidt, J. E.; Wang, R.-P.; van Ravenhorst, I. K.; Oord, R.; Chen, T.; de Groot, F.; Meirer, F.; Weckhuysen, B. M. Deactivation of Cu-Exchanged Automotive-Emission $\mathrm{NH}_{3}$-SCR Catalysts Elucidated with Nanoscale Resolution Using Scanning Transmission X-ray Microscopy. Angew. Chem. 2020, 132, 15740-15747.

(50) Czyżyk, M. T.; Sawatzky, G. A. Local-density functional and onsite correlations: The electronic structure of $\mathrm{La}_{2} \mathrm{CuO}_{4}$ and $\mathrm{LaCuO}_{3}$. Phys. Rev. B: Condens. Matter Mater. Phys. 1994, 49, 14211-14228.

(51) Mohamed, M.; May, M. M.; Kanis, M.; Brützam, M.; Uecker, R.; van de Krol, R.; Janowitz, C.; Mulazzi, M. The electronic structure and the formation of polarons in Mo-doped $\mathrm{BiVO}_{4}$ measured by angleresolved photoemission spectroscopy. RSC $A d v$. 2019, 9, 1560615614.

(52) Wiktor, J.; Ambrosio, F.; Pasquarello, A. Role of Polarons in Water Splitting: The Case of $\mathrm{BiVO}_{4}$. ACS Energy Lett. 2018, 3, 16931697. 